\title{
Metodologia ativa aplicada ao ensino odontológico: um panorama nacional a partir de um estudo bibliométrico
}

Active methodology applied to dental education: a national panorama based on a bibliometric study Metodología activa aplicada a la enseñanza odontológica: un panorama nacional a partir de un estudio bibliométrico

Monicky Mel Silva Araújo MACIEL ${ }^{1}$

Klayne Barros Nascimento da SILVA ${ }^{\mathbf{1}}$

Jéssica Gomes Alcoforado de MELO²

Diego Moura SOARES ${ }^{3}$

${ }^{1}$ Departamento de Odontologia, Uninassau, 55000-000, Caruaru-PE, Brasil

${ }_{2}^{2}$ Programa de Pós-Graduação em Odontologia, UEPB Univ. Estadual da Paraíba, 58429-500, Campina Grande-PB, Brasil

${ }^{3}$ Departamento de Odontologia, FIS Faculdade de Integração do Sertão, 56909-205, Serra Talhada-PE, Brasil

\section{Resumo}

A formação dos profissionais de saúde tem sido pautada no uso de metodologias tradicionais, entretanto, o uso da metodologia de ensino ativa tem sido uma ferramenta estimulada pelas Diretrizes Curriculares Nacionais, neste processo, os profissionais formados se tornam habilitados para desempenhar funções críticas. Este estudo tem como objetivo avaliar a produção científica nacional de trabalhos publicados nos anais do SBPqO em 2016 e 2017, que avaliaram o uso de metodologias ativas no ensino odontológico. Uma busca ativa foi realizada em todos os resumos publicados e avaliou-se aspectos como distribuição geográfica, financiamento, tipo de instituição e área da Odontologia. Foram lidos um total de 6.500 artigos mas apenas 32 foram incluídos. A região sul foi responsável pela maior quantidade dessa produção (51\%). As pesquisas realizadas nas instituições privadas representaram $45 \%$ da produção nacional e apenas $28 \%$ dos trabalhos foram financiados. Com relação a área de conhecimento a Radiologia e Cirurgia/Anestesiologia foram os mais predominantes correspondendo a $15,3 \%$ do total cada. Verificou-se uma escassez da produção científica voltada para o uso de metodologias ativas no Brasil, bem como a necessidade de descentralização dessa produção nas demais regiões/estados brasileiros e uma maior diversificação de áreas de conhecimento. Descritores: Odontologia; Educação em Odontologia; Educação em Saúde.

\section{Abstract}

The training of health professionals has been based on the use of traditional methodologies, however, the use of active teaching methodology has been a tool stimulated by the National Curricular Guidelines, in this process, trained professionals become qualified to perform critical functions. This study aims to evaluate the national scientific production of papers published in the annals of SBPqO in 2016 and 2017 , which evaluated the use of active methodologies in dental education. An active search was carried out in all published abstracts and aspects such as geographic distribution, design, type of institution and Dentistry area were evaluated. A total of 6,500 articles were read but only 32 were included. The southern region accounted for the largest quantity of this production $(51 \%)$. Research carried out in private institutions represented $45 \%$ of the national production and only $28 \%$ of the works were financed. Regarding the area of knowledge, Radiology and Surgery / Anesthesiology were the most predominant, corresponding to $15.3 \%$ of the total each. There was a shortage of scientific production aimed at the use of active methodologies in Brazil, as well as the need for decentralization of this production in other Brazilian regions / regions and a greater diversification of areas of knowledge.

Descriptors: Dentistry; Education, Dental; Health Education.

\section{Resumen}

La formación de los profesionales de la salud ha sido pautada en el uso de metodologías tradicionales, sin embargo, el uso de la metodología de enseñanza activa ha sido una herramienta estimulada por las Directrices Curriculares Nacionales, en este proceso, los profesionales formados se tornan habilitados para desempeñar funciones críticas. Este estudio tiene como objetivo evaluar la producción científica nacional de trabajos publicados en los anales del SBPqO en 2016 y 2017, que evaluaron el uso de metodologías activas en la enseñanza odontológica. Una búsqueda activa se realizó en todos los resúmenes publicados y se evaluaron aspectos como distribución geográfica, finalización, tipo de institución y área de la Odontología. Se leyeron un total de 6.500 artículos pero sólo 32 fueron incluidos. La región sur fue responsable de la mayor cantidad de esa producción (51\%). Las encuestas realizadas en las instituciones privadas representaron el $45 \%$ de la producción nacional y sólo el $28 \%$ de los trabajos fueron financiados. Con respecto al área de conocimiento la Radiología y Cirugía / Anestesiología fueron los más predominantes correspondiendo al 15,3\% del total cada uno. Se verificó una escasez de la producción científica orientada al uso de metodologías activas en Brasil, así como la necesidad de descentralización de esa producción en las demás regiones / estados brasileños y una mayor diversificación de áreas de conocimiento.

Descriptores: Odontología; Educación en Odontología; Educación en Salud.

\section{INTRODUÇÃO}

A formação dos profissionais de saúde tem sido pautada no uso de metodologias tradicionais, fragmentando o conhecimento em campos especializados $^{1}$. O processo ensino-aprendizagem se restringia apenas a reproduzir o conhecimento, sendo o docente um transmissor do conteúdo e o discente, um reprodutor, um mero expectador, sem a crítica e a reflexão ${ }^{2}$.

A Lei de Diretrizes e Bases da Educação Nacional (LDBEN) estimula o conhecimento dos problemas do mundo atual e a prestação de serviço à população, sendo reafirmada pelas Diretrizes Curriculares Nacionais (DCNs), indicando que os novos profissionais devem ser capazes de atuar no Sistema Único de Saúde (SUS) e desempenhar funções de auxílio para melhorar a qualidade de vida da população ${ }^{3}$.

Fernandes et al. $^{4}$ afirmam que o aprender a aprender na formação dos profissionais de saúde deve compreender: o aprender a conhecer, o aprender a fazer, o aprender a conviver e o aprender a ser. As metodologias ativas estão alicerçadas na autonomia ${ }^{5}$, e o ensinar exige respeito à autonomia e à dignidade de cada sujeito ${ }^{6}$. O ato de aprender é um processo que permite o estabelecimento de diferentes tipos de relações entre fatos e objetos, desencadeando ressignificações e contribuindo para a sua utilização em diferentes situações ${ }^{7}$, a partir de uma problematização como estratégia de ensinoaprendizagem .

Dessa forma, os centros de ensino devem proporcionar a formação de profissionais críticos, 
capazes de aplicar a técnica com excelência, mas também habilitados para desempenhar funções críticas. Para isso, verificou-se a necessidade da construção coletiva de um projeto pedagógico, centrado no discente como sujeito da aprendizagem e apoiado no docente como facilitador desse processo. Utilizando metodologias ativas para que o aluno seja responsável pela própria educação ${ }^{3}$.

Diante do exposto, este estudo tem como objetivo avaliar a produção científica nacional de trabalhos que buscam maneiras de aprimorar o ensino da Odontologia utilizando metodologias ativas de ensino, avaliando aspectos como distribuição geográfica da produção, financiamento, tipo de instituição, área da Odontologia.

\section{MATERIAL E MÉTODO}

O estudo foi caracterizado como sendo observacional, retrospectivo, realizado por meio da técnica de observação indireta e a análise de dados secundários. Os trabalhos apresentados na Reunião Anual da Sociedade Brasileira de Pesquisa Odontológica (SBPqO) serviram como material de pesquisa para o desenvolvimento deste artigo. Foram analisados os resumos publicados pelo encontro, considerando apenas os escritos em língua portuguesa e que buscam formas de aprimorar o ensino odontológico utilizando Metodologias Ativas de Ensino.

Embora o SBPqO possua um painel específico para tratar o tema "Educação", foi verificado que apenas alguns artigos da categoria, estavam relacionados com a temática, além dos trabalhos contidos no painel específico para "Educação" todos os demais resumos publicados nos anais da Reunião da SBPqO nos anos de 2016 e 2017 foram analisados. Para efeito desta pesquisa, apenas foram incluídos os trabalhos que abordavam temas a respeito de propostas e experiências de técnicas de metodologias ativas aplicadas ao ensino nos cursos de Odontologia. Foram descartados, sumariamente, os artigos que, embora tivessem relação com educação, pesquisavam avaliação curricular, gestão educacional e temas que não tivessem relação com metodologias ativas de ensino.

Os dados foram coletados nos anais das $33^{\mathrm{a}} \mathrm{e}$ $34^{\mathrm{a}}$ Reunião Anual da SBPqO, realizadas em 2016 e 2017, na cidade de Campinas, São Paulo, a partir de busca manual com a leitura na integra de todos os resumos, sendo realizada por dois pesquisadores devidamente calibrados. A escolha pelos anais do SBPqO foi motivada pela relevância da Reunião Anual para o desenvolvimento da pesquisa científica no país, já que o encontro reune trabalhos desenvolvidos por acadêmicos, pós-graduandos, professores e pesquisadores ${ }^{9,10}$.

Para a catalogação dos dados foi desenvolvida uma planilha própria, no programa
Excel 2013, versão Professional Plus. Os dados analisados foram relativos à instituição onde o trabalho foi desenvolvido, estado e região do país (Centro-oeste, Nordeste, Norte, Sudeste e Sul); se a instituição é privada, Estadual ou Federal; se houve financiamento; área de conhecimento do estudo (cirurgia/anestesiologia, periodontia, prótese, dentística/cariologia, Odontologia social/saúde coletiva, dentre outros) e, por fim, o tipo do trabalho se revisão de literatura, relato de experiência ou pesquisa.

Para a análise dos dados, após os mesmos terem sido tabulados no Excel 2013, versão Professional Plus, foram analisados com o auxílio do software SPSS na versão 16 e apresentados por meio da estatística descritiva (frequências absolutas e percentual).

\section{RESULTADOS}

Foram lidos um total de 6.500 artigos publicados nos anais das Reuniões da Sociedade Brasileira de Pesquisa Odontológica dos anos de 2016 e $2017^{11,12}$. Deste universo, apenas 32 trabalhos tratavam sobre metodologias ativas de ensino para o curso de Odontologia. Dos 32 resumos que compuseram a amostra, 20 deles foram publicados nos anais da reunião de 2017 e 12 na reunião de 2016. Em se tratando do tipo de trabalho, 94\% (30 resumos) retratavam trabalhos de pesquisa e apenas 6\% (dois trabalhos) eram de revisão de literatura.

Com relação a distribuição dos trabalhos de acordo com a região do país, a região Sul foi responsável por $51 \%$ (16 resumos) da produção nacional, seguida pela região Sudeste com 42,6\% (13 trabalhos). Dentre os estados da região Sul, o Paraná é responsável por $32 \%$ da produção do país. Não foi encontrada nenhuma produção desenvolvida na regisão centro-oeste brasileira. A distribuição da produção de acordo com as regiões e estados brasileiros pode ser vista na Tabela 1.

A Tabela 2 mostra a distribuição dos trabalhos de acordo com o tipo de instituição (federal, estadual e privada) e a região do país. As pesquisas realizadas nas instituições privadas representaram $45 \%$ da produção nacional, estando a maioria delas concentradas na região Sul. Em se tratando do recebimento de fomento à pesquisa, apenas $28 \%$ dos trabalhos foram financiados. Os financiamentos advindos das Instituições de Ensino Superior (IES) corresponderam a $50 \%$ do total, seguido do Conselho Nacional de Desenvolvimento Científico e Tecnológico (CNPq) e das Fundações de Amparo a Pesquisa (FAPs) com $20 \%$ cada (Tabela 3 ).

No tocante a área de conhecimento dos trabalhos, verificou-se que os estudos que relacionavam metodologias ativas aplicadas as áreas de Radiologia e Cirurgia/Anestesiologia foram os mais predominantes correspondendo a $15,3 \%$ do total 
cada uma das duas áreas referida, seguido de Odontologia Social/Saúde Coletiva com 12,5 e Dentística/Cariologia com 9,3\%.

Tabela 1. Distribuição dos trabalhos de acordo com a região e o estado brasileiro

\begin{tabular}{ccc}
\hline Região/Estado & $\begin{array}{c}\text { Frequência } \\
\text { Absoluta } \\
\text { (n) }\end{array}$ & $\begin{array}{c}\text { Frequência } \\
\text { relativa } \\
\text { (\%) }\end{array}$ \\
\hline NORDESTE & $\mathbf{1}$ & $\mathbf{3 , 2 \%}$ \\
Ceará & 1 & $3,2 \%$ \\
\hline NORTE & $\mathbf{1}$ & $\mathbf{3 , 2 \%}$ \\
Pará & 1 & $3,2 \%$ \\
\hline SUDESTE & $\mathbf{1 3}$ & $\mathbf{4 2 , 6 \%}$ \\
Rio de Janeiro & 2 & $6,4 \%$ \\
São Paulo & 10 & $33,0 \%$ \\
Minas Gerais & 1 & $3,2 \%$ \\
\hline SUL $\quad \mathbf{1 6}$ & $\mathbf{5 1 , 0 \%}$ \\
Paraná & 10 & $32,0 \%$ \\
Rio Grande do Sul & 6 & $19,0 \%$ \\
\hline Total & $\mathbf{3 2}$ & $\mathbf{1 0 0 \%}$ \\
\hline
\end{tabular}

Tabela 2. Distribuição dos trabalhos de acordo com o tipo de instituição (federal, estadual e privada) e a região do país

\begin{tabular}{ccc}
\hline Instituição & $\begin{array}{c}\text { Frequência } \\
\text { Absoluta } \\
\text { (n) }\end{array}$ & $\begin{array}{c}\text { Frequência } \\
\text { Relativa } \\
\text { (\%) }\end{array}$ \\
\hline FEDERAL & $\mathbf{1 0}$ & $\mathbf{3 0 , 0 \%}$ \\
Nordeste & 1 & $3,0 \%$ \\
Norte & 1 & $3,0 \%$ \\
Sudeste & 1 & $3,0 \%$ \\
Sul & 7 & $21,0 \%$ \\
\hline ESTADUAL & 7 & $\mathbf{2 5 , 0 \%}$ \\
Sudeste & 6 & $\mathbf{2 1 , 0 \%}$ \\
Sul & 1 & $4,0 \%$ \\
\hline PRIVADA & $\mathbf{1 5}$ & $\mathbf{4 5 , 0 \%}$ \\
Centro-Oeste & 1 & 2,4 \\
Nordeste & 1 & 2,4 \\
Sudeste & 5 & $\mathbf{2 2 , 2}$ \\
Sul & 8 & $\mathbf{1 8 , 0}$ \\
\hline Total & $\mathbf{3 2}$ & $\mathbf{1 0 0 \%}$ \\
\hline
\end{tabular}

Tabela 3. Distribuição dos trabalhos de acordo com o recebimento de fomento (agência financiadora) e a região do país

\begin{tabular}{|c|c|c|}
\hline Fomento & $\begin{array}{c}\text { Frequência } \\
\text { Absoluta } \\
\text { (n) }\end{array}$ & $\begin{array}{c}\text { Frequência } \\
\text { Relativa } \\
\text { (\%) }\end{array}$ \\
\hline CNPQ & 2 & $\mathbf{2 0}, \mathbf{0} \%$ \\
\hline Norte & 1 & $10,0 \%$ \\
\hline Sudeste & 1 & $10,0 \%$ \\
\hline FAPs & 2 & $20,0 \%$ \\
\hline Sudeste & 1 & $10,0 \%$ \\
\hline Sul & 1 & $10,0 \%$ \\
\hline IES & 5 & $50,0 \%$ \\
\hline Sudeste & 5 & $50,0 \%$ \\
\hline OUTROS* $^{*}$ & $\mathbf{1}$ & $10,0 \%$ \\
\hline Sudeste & 1 & $10,0 \%$ \\
\hline Total & 9 & $100,0 \%$ \\
\hline
\end{tabular}

Na Tabela 4 pode ser verificada de forma detalhada a distribuição dos trabalhos de acordo com a área de conhecimento.

Tabela 4. Distribuição dos trabalhos de acordo com a área de conhecimento

\begin{tabular}{lcc}
\hline Área de Conhecimento & $\begin{array}{c}\text { Frequência } \\
\text { Absoluta } \\
\text { (n) }\end{array}$ & $\begin{array}{c}\text { Frequência } \\
\text { relativa } \\
\text { (\%) }\end{array}$ \\
\hline Área básica & 2 & $6,25 \%$ \\
\hline Cirurgia / Anestesiologia & 5 & $15,3 \%$ \\
\hline Dentistica / Cariologia & 3 & $9,30 \%$ \\
\hline Estomatologia & 2 & $6,25 \%$ \\
\hline Odontologia Hospitalar & 1 & $3,10 \%$ \\
\hline Odontologia Social/ & 4 & $12,5 \%$ \\
\hline Saúde Coletiva & 2 & $6,25 \%$ \\
\hline Odontopediatria & 2 & $6,25 \%$ \\
\hline Pacientes Especiais & 2 & $6,25 \%$ \\
\hline Periodontia & 2 & $6,25 \%$ \\
\hline Prótese & 5 & $15,6 \%$ \\
\hline Radiologia & 2 & $6,25 \%$ \\
\hline${ }^{*}$ Outros & $\mathbf{3 2}$ & $\mathbf{1 0 0 \%}$ \\
\hline \multicolumn{1}{c}{ Total } &
\end{tabular}

\section{DISCUSSÃO}

O processo de ensino-aprendizagem vem sendo modificado para uma abordagem centrada no aluno e uma aprendizagem baseada em competências $^{13}$. As mudanças das práticas pedagógicas estão em processo de modificação devido à Lei de Diretrizes e Bases da Educação Nacional e das Diretrizes Curriculares, que afirmam a importância e necessidade do atendimento às demandas sociais, estabelecendo assim, uma relação de reciprocidade ${ }^{14-16}$, e com isso, formar profissionais ativos e motivados a "aprender a aprender"

Entretanto, ainda são poucas as pesquisas científicas que buscam métodos para melhorar o ensino ativo e torná-lo eficiente e presente no dia-adia do estudante de Odontologia ${ }^{18}$. Fato que é confirmado diante do número reduzido de trabalhos (32 de 6.500 artigos) que pesquisaram sobre o uso das metodologias ativas nas universidades brasileiras.

A produção científica no Brasil está concentrada na região Sudeste, onde São Paulo, Rio de Janeiro e Minas Gerais são os estados que mais se destacam nesse cenário ${ }^{19}$, essa afirmativa é corroborada pelos resultados observados no estudo de Cavalcanti et al. ${ }^{9}$. O que difere dos achados deste estudo, visto que a região Sul foi predominante na produção científica sobre a utilização de metodologias ativas, em especial o estado do Paraná com $32 \%$ da produção do país. Tal discrepância, na produção das regiões do país, pode ser atribuída à produção de conhecimento científico que se concentra na região que necessita de tal produção ${ }^{20}$. 
Buscando avaliar a produção científica sobre aprendizagem baseada em problema (ABP) em periódicos de referência em educação médica no Brasil e no mundo Cavalcante et al. ${ }^{21}$ verificaram que os estudos nacionais sobre o tema concentram-se nos estados da região Sul e Sudeste, o que corroboram com os dados encontrados neste artigo. Os autores ainda sugerem que a produção científica sobre ABP no Brasil ainda é bastante escassa e que ainda assim, resultados positivos desse tipo de metodologia ativa na formação médica e na prática profissional foram encontrados. Da mesma forma verificou-se uma grande escassez de trabalhos utilizando não apenas $\mathrm{ABP}$, mas como outros métodos ativos de ensino para os cursos de Odontologia. Os autores reforçam ainda a necessidade de mais estudos a respeito do método para que maiores conclusões possam ser formuladas.

Além da concentração geográfica, a atividade de pesquisa no Brasil é predominantemente acadêmica, em instituições que ministram cursos de graduação e pós-graduação ${ }^{22}$. Neste estudo, as instituições privadas representaram $45 \%$ da produção nacional, e grande parte está concentrada na região Sul.

Os processos de mudanças tendem a se manifestar de modo mais rápido nas instituições privadas, pois elas tendem a se adequar às novas normas, diferente das instituições públicas onde o processo é mais desarticulado e lento. Tal fato seria devido aos professores serem mais jovens, caracterizando-se por experiências e motivações diferentes no que se refere ao enfrentamento das mudanças ${ }^{23}$.

A expansão da produção científica promoveu um aumento expressivo na demanda por recursos de financiamento ${ }^{9}$. As agências de fomento federais com maior presença em financiamento são o $\mathrm{CNPq}$ e a Coordenação de Aperfeiçoamento de Pessoal de Nível Superior (Capes), além das agências estaduais de fomento que são as Fundações de Amparo à Pesquisa (FAPs). Nos últimos anos, a política geral de financiamento para o setor público do governo Federal é a de diminuir o desembolso ${ }^{22}$.

Mesmo o financiamento se mostrando de grande importância, ele se apresenta insuficiente ${ }^{10}$, conforme os achados deste estudo, que apontam que apenas $28 \%$ dos trabalhos foram financiados por agências de fomento. Dentre eles, as Instituições de Ensino Superior foram responsáveis por 50\%, seguido do CNPq e das FAPs, que corresponderam a $20 \%$ cada.

As áreas que sempre despertaram o interesse dos cirurgiões-dentistas são as que predominam, neste estudo no uso das metodologias ativas: Radiologia e Cirurgia/Anestesiologia, com 15,3\% do total em cada uma das áreas. Devido à mudança na atuação do profissional frente à população, em que se está preocupado em prevenir e promover saúde à apenas tratar uma lesão ${ }^{24}$, as áreas da Odontologia Social/Saúde Coletiva se encontram em segundo lugar no uso das metodologias ativas, com 12,5\%.

Melo et al. ${ }^{25}$ realizaram uma busca nos anais das reuniões da SBPqO dos anos de 2007 a 2016, utilizando metodologia semelhante a deste artigo, com o objetivo de avaliar a produção científica nacional em odontologia a respeito da aprendizagem baseada em problema (APB). Foram encontrados apenas 12 resumos distribuídos em todos os anos analisados. Isso reforça os resultados verificados neste artigo, com relação a escassez de pesquisas sobre o tema. Além disso, de acordo com os mesmos autores, apenas um dos 12 trabalhos incluídos recebeu auxílio financeiro. $\mathrm{O}$ que corrobora com os resultados desta pesquisa em relação ao pouco financiamento destinado a pesquisas com está temática.

\section{CONCLUSÃO}

Diante do exposto, verifica-se a escassez da produção científica voltada para o uso de metodologias ativas no Brasil, bem como a necessidade de descentralização dessa produção nas demais regiões/estados brasileiros. Uma maior diversificação de áreas de conhecimento, utilizando métodos ativos de ensino em Odontologia, deve ser estimulado, visto que a produção dos últimos dois anos se concentra prioritariamente nas áreas de Radiologia e Cirurgia/Anestesiologia. Também se faz necessário um maior estímulo às instituições públicas, principalmente as estaduais, em pesquisarem a eficácia do uso dessas metodologias. Uma maior parcela de financiamento pelos órgãos de fomento estaduais e federais deve ser estimulada.

\section{REFERÊNCIAS}

1. Capra F. O ponto da mutação: a ciência, a sociedade e a cultura emergente. São Paulo: Cultrix; 2006.

2. Behrens MA. O paradigma emergente e a prática pedagógica. Petrópolis: Vozes; 2005.

3. Brasil MEC. Diretrizes curriculares nacionais para os cursos de graduação em odontologia. Diário Oficial da União, 2002.[Acesso em 10 de janeiro de 2018]; Resolução CNE/CES 3/2002. Seção 1, p. 10. Disponível em: http://portal.mec.gov.br/ cne/arquivos/pdf/ CES032002.pdf

4. Fernandes JD, Ferreira SL, La Torre MPS, Rosa DOS, Costa HOG. Diretrizes estratégicas para a implantação de uma nova proposta pedagógica na Escola de Enfermagem da Universidade da Federal da Bahia. Rev bras enferm. 2003;56(54):392-95.

5. Freire P. Pedagogia da autonomia: saberes necessários à prática educativa. 33. ed. São Paulo: Paz e Terra; 2006.

6. Costa CRBSF, Siqueira-Batista R. As teorias do desenvolvimento moral e o ensino médico: uma 
reflexão pedagógica centrada na autonomia do educando. Rev Bras Educ Med. 2004;28(3):242-50.

7. Demo P. Professor do futuro e reconstrução do conhecimento. Petrópolis: Vozes; 2004.

8. Cyrino EG, Toralles-Pereira ML. Trabalhando com estratégias de ensino-aprendizado por descoberta na área da saúde: a problematização e a aprendizagem baseada em problemas. Cad Saúde Pública. 2004;20(3):780-88.

9. Cavalcanti AL, Melo TRNB, Barrosos KMA, Souza FEC, Maia AMA, Silva ALO. Perfil da Pesquisa Científica em Odontologia Realizada no Brasil. Pesqui bras odontopediatria clin integr. 2004;4(2):99-104.

10. Xavier AFC, Silva ALO, Cavalcanti AL. Análise da produção científica em Odontologia no nordeste brasileiro com base em um congresso odontológico. Arq Odontol. 2011;47(3):127-34.

11. SBPqO. Estatística SBPqO. Conexão SBPqO, 2016 [Acesso em 03 de janeiro de 2018];30 (supl 1). Disponível em: http://www.sbpqo.org.br/ hotsite2016/anais/BOR-v030-Suppl-Book-p7d.pdf

12. SBPqO. Estatística SBPqO. Conexão SBPqO. [Acesso em 03 de janeiro de 2018]; 31(supl. 2). Disponível em: http://www.sbpqo.org.br/ hotsite 2017/Anais/BOR-v031-SBPqO-Book_v12.pdf

13. Oliver R, Kersten H, Vinkka-Puhakka H, Alpasan G, Bearn D, Cema I et al. Curriculum structure: principles and strategy. Eur $J$ Dent Educ. 2008;12(Suppl 1):74-84.

14. Brasil. Lei no 9.394 de 20 de dezembro de 1996. Diário Oficial da União 1996.

15. Cotta RMM, Mendes FF, Muniz JN. Descentralização das políticas públicas de saúde do imaginário ao real. Viçosa: UFV/Cebes; 1998.

16. Almeida M. Diretrizes curriculares para os cursos universitários na área de saúde. Londrina: Rede Unida; 2003.

17. Mitre SM, Siqueira-Batista R, Girardi-deMendonça JM, Morais-Pinto NM, Meirelles CAB, Pinto-Porto $\mathrm{C}$ et al. Metodologias ativas de ensino-aprendizagem na formação profissional em saúde: debates atuais. Ciên. Saúde Coletiva. 2008;13(Sup 2):2133-44.

18. Carvalho RB, Costa TBC, Gomes MJ, Santos KT, Guerra SMG. Formação docente em odontologia no Brasil: sugestões de mudanças após as diretrizes curriculares nacionais. Rev Bras Pesqui Saúde; 2010;12(4):39-44.

19. Aquino SN, Martelli DR, Bonan PRF, Laranjeira AL, Martelli Júnior $H$. Produção científica odontológica e relação com agências de financiamento de pesquisa. Arq Odontol. 2009;45(3):142-46.

20. Araújo EC, Batista SH, Gerab IF. A Produção Científica sobre Docência em Saúde: um Estudo em Periódicos Nacionais. Rev bras educ med. 2011;35(4):486-92.
21. Cavalcante AN, Lira GV, Cavalcante Neto PG, Lira RCM. Análise da Produção Bibliográfica sobre Problem-Based Learning (PBL) em Quatro Periódicos Selecionados. Rev bras educ med. 2018;42(1):15-26.

22. Guimarães R, Lourenço R, Cosac S. A pesquisa em epidemiologia no Brasil. Rev Saúde Pública. 2001;35(4):321-40.

23. Secco LG, Pereira MLT. Formadores em odontologia:profissionalização docente e desafios político-estruturais. Ciênc saúde coletiva. 2004;9(1):113-20.

24. Dias AA, Narvai PC, Rego DM. Tendências da produção científica em odontologia no Brasil. Rev Panam Salud Publica/Pan Am J Public Health 2008;24(1):54-60.

25. Melo NB, Fernandes Neto JA, Catão MHCV, Bento PM. Metodologia da problematização e aprendizagem baseada em problemas na odontologia: análise bibliométrica dos trabalhos apresentados nas Reuniões da SBPqO. Rev ABENO. 2017;17(2):60-7.

\section{CONFLITO DE INTERESSES}

Os autores declaram não haver conflitos de interesse.

\section{AUTOR PARA CORRESPONDENCIA}

\section{Diego Moura Soares}

diegomsoares@hotmail.com

Submetido em 23/08/2018 Aceito em 04/10/2018 